\title{
La terapia ocupacional mejora las actividades personales de la vida diaria de quienes sufrieron un accidente cerebro-vascular
}

Occupational therapy improves personal activities of daily living after stroke

\section{Objetivo}

Determinar si la terapia ocupacional enfocada específicamente en las actividades personales de la vida diaria (TOAVD) mejora la recuperación de los pacientes que sufrieron un accidente cerebro-vascular (ACV).

\section{Diseño}

Revisión sistemática.

\section{Fuente y selección de datos}

Registro de ensayos del grupo de Stroke Cochrane, CENTRAL, MEDLINE, EMBASE, CINAHL, PsycLIT, AMED, Wilson Social Sciences Abstracts, Science Citation Index, Social Science Citation Index y Arts and humnities Citation index; trabajos no publicados, estudios en curso y lista de referencias. Se contacto a autores e investigadores y se realizó una búsqueda manual. Se identificaron ensayos clínicos controlados que contaran con una definición clínica de ACV y cuyos pacientes de la rama "intervención" hubieran recibido TOAVD provista por un te-rapista ocupacional calificado. Dos revisores independientes seleccionaron los trabajos y extrajeron los resultados a reportar. Los resultados primarios fueron la proporción de pacientes que se deterioraba o era dependiente en las actividades personales al final del período de seguimiento, y el rendimiento en las AVD.

\section{Resultados principales}

Fueron seleccionados nueve estudios (1.258 participantes de 55 a 87,5 años) con una proporción de sexo masculino de 19 a $66 \%$. Todos habían excluido a los individuos con dificultades en la comunicación, trastornos cognitivos $u$ otras dificultades que interfirieran con la intervención (ej. enfermedad terminal o imposibilidad para comunicarse en inglés). El tiempo medio de seguimiento fue seis meses. La TOAVD luego de haber sufrido un ACV mejoró los puntajes de rendimiento en las AVD con una diferencia media estandarizada (DME) de 0,18 (IC95\% 0,04 a 0,32 ) y se asoció a una disminución del riesgo un resultado adverso combinado (muerte, deterioro o dependencia) en las AVD (OR 0,67; IC95\% 0,51 a 0,87).

\section{Conclusiones}

Los pacientes que reciben TOAVD posteriormente a un ACV presentan menor probabilidad de deteriorarse y mayor probabilidad de realizar las AVD de forma independiente. Sin embargo, la naturaleza exacta de la TOAVD como intervención necesita ser definida.

Palabras clave: accidente cerebrovascular, terapia ocupacional, actividades de la vida diaria.

Key words: stroke, occupational therapy, activities of daily living.

Fuente de financiamiento: Cochrane, Big lottery Fund y Chest Heart and Stroke Scotland.

\section{Comentario}

En esta revisión se observó que la TOAVD es una intervención efectiva no farmacológica que permitiría mejorar las AVD de pacientes que han sufrido un $A C V$, requiriendo que aproximadamente 11 pacientes (IC95\% 7 a 30 ) sean sometidos a la misma para evitar que uno de ellos deteriore sus AVD.

Vale aclarar que cuando los autores se refieren a diferencias medias estandarizadas (DME), una DME de 0,18 equivale a un punto de diferencia en la escala de Barthel, lo que implica que el paciente haya logrado realizar en forma independiente una actividad que antes no podía hacer, como comer o vestirse. Revisiones sistemáticas previas sobre terapias de rehabilitación ${ }^{1,2,3}$ habían observado que los pacientes mejoraban en sus AVD, pero esta es la primera que enfoca específicamente en la TOAVD. Si bien la búsqueda fue exhaustiva y la metodología resulta apropiada, podemos suponer algunas dificultades en el enmascaramiento de la intervención, lo que puede conducir a sesgos. Por ejemplo, es imposible en estos casos que la intervención sea totalmente "ciega" y muy difícil que lo sea la evaluación de los resultados, especialmente teniendo en cuenta que el mismo terapista ocupacional suele ser el investigador.

Un aspecto importante en la mejoría de la calidad de vida post ACV es encontrar la mejor estrategia de prevención secundaria maximizando el esfuerzo para mejorar la calidad funcional. En este sentido, sería útil establecer durante cuanto tiempo y con qué intensidad es necesario realizar este tipo de intervención.

\section{Conclusiones de la comentadora}

Siendo el ACV la segunda causa de muerte en el mundo y la primera causa de discapacidad a largo plazo en adultos, y teniendo en cuenta que la mitad de los pacientes que sobreviven a un ACV queda con discapacidad para desarrollar sus actividades personales, es prioritario establecer y ponderar las intervenciones que puedan beneficiarlos.

Marina Romano [ Servicio de Neurología del Hospital Italiano de Buenos Aires. marina.romano@ hospitalitaliano.org.ar ]

Romano M. La terapia ocupacional mejora las actividades personales de la vida diaria de quienes sufrieron un accidente cerebro-vascular. Evid. actual. práct. ambul; 11(4):110, Jul-Ago.2008. Comentado de: Legg $L$, et al. Occupational therapy for patients with problems in personal activities of daily living after stroke: systematic review of randomized trials. BMJ.2007 Nov 3;335(7626):922. PMID: 17901469.

Disponible en URL: http://www.bmj.com/cgi/reprint/335/7626/922 (último acceso 29/07/08).

\section{Referencia}

1. Legg L y col. Occupational therapy for patients with problems in activities of daily living after stroke. Cochrane Database Syst Rev. 2006 Oct $18 ;(4): C D 003585$.

2. Legg $L$ y col. Outpatient Service Trialists. Rehabilitation therapy services for stroke patients living at home: systematic review of randomised trials Lancet. 2004 Jan 31 ; 363(9406):352-6.

3. Govender P. Benefits of occupational therapy in stroke rehabilitation. Expert Rev Neurother. 2007 Aug; 7(8):1013-9. 